\title{
On the Complexity and Decidability of Some Problems Involving Shuffle ${ }^{\text {tr }}$
}

\author{
Joey Eremondi ${ }^{\mathrm{a}, 2}$, Oscar H. Ibarra ${ }^{\mathrm{b}, 1}$, Ian McQuillan ${ }^{\mathrm{c}, 2, *}$ \\ ${ }^{a}$ Department of Information and Computing Sciences \\ Utrecht University, P.O. Box 80.0893508 TB Utrecht, The Netherlands \\ ${ }^{b}$ Department of Computer Science \\ University of California, Santa Barbara, CA 93106, USA \\ ${ }^{c}$ Department of Computer Science, University of Saskatchewan \\ Saskatoon, SK S7N 5A9, Canada
}

\begin{abstract}
The complexity and decidability of various decision problems involving the shuffle operation (denoted by $ш$ ) are studied. The following three problems are all shown to be NP-complete: given a nondeterministic finite automaton (NFA) $M$, and two words $u$ and $v$, is $L(M) \nsubseteq u ш v$, is $u ш v \nsubseteq L(M)$, and is $L(M) \neq u ш v$ ? It is also shown that there is a polynomial-time algorithm to determine, for NFAs $M_{1}, M_{2}$, and a deterministic pushdown automaton $M_{3}$, whether $L\left(M_{1}\right) ш L\left(M_{2}\right) \subseteq L\left(M_{3}\right)$. The same is true when $M_{1}, M_{2}, M_{3}$ are one-way nondeterministic $l$-reversal-bounded $k$-counter machines, with $M_{3}$ being deterministic. Other decidability and complexity results are presented for testing whether given languages $L_{1}, L_{2}$, and $R$ from various languages families satisfy $L_{1} ш L_{2} \subseteq R$, and $R \subseteq L_{1} ш L_{2}$. Several closure results on shuffle are also shown.
\end{abstract}

Keywords: Automata and Logic, Shuffle, Counter Machines, Pushdown Machines, Reversal-Bounds, Determinism, Commutativity, Strings

\section{Introduction}

The shuffle operator models the natural interleaving between strings. It was introduced by Ginsburg and Spanier [1], where it was shown that context-free languages are closed under shuffle with regular languages, but not context-free languages. It has since been applied in a number of areas such as concurrency [2], coding theory [3], verification [4], database schema [5], and biocomputing [3, 6], and has also received considerable study in the area of formal languages. However, there remains a number of open questions, such as the long-standing problem as to whether it is decidable, given a regular language $R$ to tell if $R$ has a non-trivial decomposition; that is, $R=L_{1} ш L_{2}$, for some $L_{1}, L_{2}$ that are not the language consisting of only the empty word [7].

This paper addresses several complexity-theoretic and decidability questions involving shuffle. In the past, similar questions have been studied by Ogden, Riddle, and Round [2], who showed that there exists deterministic context-free languages $L_{1}, L_{2}$ where $L_{1} ш L_{2}$ is NP-complete. More recently, L. Kari studied problems involving solutions to language equations of the form $R=L_{1} ш L_{2}$, where some of $R, L_{1}, L_{2}$ are given, and the goal is to determine a procedure, or determine that none exists, to solve for the variable(s) [8]. Also, there has been similar decidability problems investigated involving shuffle on trajectories [9], where

\footnotetext{
(C)2016. This manuscript version is made available under the CC-BY-NC-ND 4.0 license http://creativecommons.org/licenses/by-nc-nd/4.0/

*Corresponding author

URL: j.s.eremondi@students.uu.nl (Joey Eremondi), ibarra@cs.ucsb.edu (Oscar H. Ibarra), mcquillan@cs.usask.ca (Ian McQuillan)

${ }^{1}$ Supported, in part, by NSF Grant CCF-1117708 (Oscar H. Ibarra).

${ }^{2}$ Supported, in part, by Natural Sciences and Engineering Research Council of Canada Grant 327486-2010 (Ian McQuillan).
} 
the patterns of interleaving are restricting according to another language $T \subseteq\{0,1\}^{*}$ (a zero indicates that a letter from the first operand will be chosen next, and a one indicates a letter from the second operand is chosen). L. Kari and Sosík show that it is decidable, given $L_{1}, L_{2}, R$ as regular languages with a regular trajectory set $T$, whether $R=L_{1} \uplus_{T} L_{2}$ (the shuffle of $L_{1}$ and $L_{2}$ with trajectory set $T$ ). Furthermore, if $L_{1}$ is allowed to be context-free, then the problem becomes undecidable as long as, for every $n \in \mathbb{N}$, there is some word of $T$ with more than $n$ 0's (with a symmetric result if there is a context-free language on the right). This implies that it is undecidable whether $L_{1} ш L_{2}=R$, where $R$ and one of $L_{1}, L_{2}$ are regular, and the other is context-free. In [10], it is demonstrated that given two linear context-free languages, it is not semi-decidable whether their shuffle is linear context-free, and given two deterministic context-free languages, it is not semi-decidable whether their shuffle is deterministic context-free. Complexity questions involving so-called shuffle languages, which are augmented from regular expressions by shuffle and iterated shuffle, have also been studied [11]. It has also been determined that it is NP-hard to determine if a given string is the shuffle of two identical strings (independently in [12] and [13]).

Recently, there have been several papers involving the shuffle of two words. It was shown that the shuffle of two words with at least two letters has a unique decomposition into the shuffle of words [14]. In fact, the shuffle of two words, each with at least two letters, has a unique decomposition over arbitrary sets of words 15]. Also, a polynomial-time algorithm has been developed that, given a deterministic finite automaton (DFA) $M$ and two words $u, v$, can test if $u ш v \subseteq L(M)$ [16. In the same work, an algorithm was presented that takes a DFA $M$ as input and outputs a "candidate solution" $u, v$; this means, if $L(M)$ has a decomposition into the shuffle of two words, $u$ and $v$ must be those two unique words. But the algorithm cannot guarantee that $L(M)$ has a decomposition. This algorithm runs in $O(|u|+|v|)$ time, which is often far less than the size of the input DFA, as DFAs accepting the shuffle of two words can be exponentially larger than the words [17]. It has also been shown [18] that the following problem is NP-complete: given a DFA $M$ and two words $u, v$, is it true that $L(M) \nsubseteq u ш v ?$

In this paper, problems are investigated involving three given languages $R, L_{1}, L_{2}$, and the goal is to determine decidability and complexity of testing if $R \nsubseteq L_{1} ш L_{2}, L_{1} ш L_{2} \nsubseteq R$, and $L_{1} ш L_{2} \neq R$, depending on the language families of $L_{1}, L_{2}$ and $R$. In Section 3 , it is demonstrated that the following three problems are NP-complete: to determine, given an NFA $M$ and two words $u, v$ whether $u ш v \nsubseteq L(M)$ is true, $L(M) \nsubseteq u ш v$ is true, and $u ш v \neq L(M)$ is true. Then, the DFA algorithm from [16] that can output a "candidate solution" is extended to an algorithm on NFAs that operates in polynomial time, and outputs two words $u, v$ such that if the NFA is decomposable into the shuffle of words, then $u ш v$ is the unique solution. And in Section 4 decidability and the complexity of testing if $L_{1} ш L_{2} \subseteq R$ is investigated involving more general language families. In particular, it is shown that it is decidable in polynomial time, given NFAs $M_{1}, M_{2}$ and a deterministic pushdown automaton $M_{3}$, whether $L\left(M_{1}\right) ш L\left(M_{2}\right) \subseteq L\left(M_{3}\right)$. The same is true given $M_{1}, M_{2}$ that are one-way nondeterministic $l$-reversal-bounded $k$-counter machines, and $M_{3}$ is a one-way deterministic $l$-reversal-bounded $k$-counter machine. However, if $M_{3}$ is a nondeterministic 1-counter machine that makes only one reversal on the counter, and $M_{1}$ and $M_{2}$ are fixed DFAs accepting $a^{*}$ and $b^{*}$ respectively, then the question is undecidable. Also, if we have fixed languages $L_{1}=(a+b)^{*}$ and $L_{2}=\{\lambda\}$, and $M_{3}$ is an NFA, then testing whether $L_{1} ш L_{2} \not \subset L\left(M_{3}\right)$ is PSPACE-complete. Also, testing whether $a^{*} ш\{\lambda\} \nsubseteq L$ is NP-complete for $L$ accepted by an NFA. For finite languages $L_{1}, L_{2}$, and $L_{3}$ accepted by an NPDA, it is NP-complete to determine if $L_{1} ш L_{2} \nsubseteq L_{3}$. Results on unary languages are also provided. In Section 5 testing $R \subseteq L_{1} ш L_{2}$ is addressed. This is already undecidable if $R$ and $L_{1}$ are deterministic pushdown automata. However, it is decidable if $L_{1}, L_{2}$ are any commutative, semilinear languages, and $R$ is a context-free language (even if augmented by reversal-bounded counters). Then, in Section [6, several other decision problems, and some closure properties of shuffle are investigated.

\section{Preliminaries}

We assume an introductory background in formal language theory and automata [19], as well as computational complexity [20]. We assume knowledge of pushdown automata, finite automata, and Turing machines, and we use notation from [19]. Let $\Sigma=\left\{a_{1}, \ldots, a_{m}\right\}$ be a finite alphabet. Then $\Sigma^{*}\left(\Sigma^{+}\right)$is the set of all (non-empty) words over $\Sigma$. A language over $\Sigma$ is any $L \subseteq \Sigma^{*}$. Given a language $L \subseteq \Sigma^{*}$, the 
complement of $L, \bar{L}=\Sigma^{*}-L$. The length of a word $w \in \Sigma^{*}$ is $|w|$, and for $a \in \Sigma,|w|_{a}$ is the number of $a$ 's in $w$.

Let $\mathbb{N}$ be the positive integers, and $\mathbb{N}_{0}$ be the non-negative integers. For $n \in \mathbb{N}_{0}$, then define $\pi(n)$ to be 0 if $n=0$, and 1 otherwise.

Next, we formally define reversal-bounded counter machines 21. A one-way $k$-counter machine is a tuple $M=\left(k, Q, \Sigma, \triangleleft, \delta, q_{0}, F\right)$, where $Q, \Sigma, \triangleleft, q_{0}, F$ are respectively, the finite set of states, input alphabet, right input end-marker (not in $\Sigma$ ), the initial state, and the set of final states. The transition function $\delta$ is a relation from $Q \times(\Sigma \cup\{\triangleleft\}) \times\{0,1\}^{k}$ into $Q \times\{\mathrm{S}, \mathrm{R}\} \times\{-1,0,+1\}^{k}$, such that if $\delta\left(q, a, c_{1}, \ldots, c_{k}\right)$ contains $\left(p, d, d_{1}, \ldots, d_{k}\right)$ and $c_{i}=0$ for some $i$, then $d_{i} \geq 0$ (this is to prevent negative values in any counter). The symbols $\mathrm{S}$ and $\mathrm{R}$ give the direction of the input tape head, being either stay or right respectively. Furthermore, $M$ is deterministic if $\delta$ is a partial function. A configuration of $M$ is a tuple $\left(q, w, c_{1}, \ldots, c_{k}\right)$ indicating that $M$ is in state $q$ with $w$ (in $\Sigma^{*}$ or $\Sigma^{*} \triangleleft$ ) as the remaining input, and $c_{1}, \ldots, c_{k} \in \mathbb{N}_{0}$ are the contents of the counters. The derivation relation $\vdash_{M}$ is defined by, $\left(q, a w, c_{1}, \ldots, c_{k}\right) \vdash_{M}\left(p, w^{\prime}, c_{1}+\right.$ $\left.d_{1}, \ldots, c_{k}+d_{k}\right)$, if $\left(p, d, d_{1}, \ldots, d_{k}\right) \in \delta\left(q, a, \pi\left(c_{1}\right), \ldots, \pi\left(c_{k}\right)\right)$ where $d=\mathrm{S}$ implies $w^{\prime}=a w$, and $d=\mathrm{R}$ implies $w^{\prime}=w$. Then $\vdash_{M}^{*}$ is the reflexive, transitive closure of $\vdash_{M}$. A word $w \in \Sigma^{*}$ is accepted by $M$ if $\left(q_{0}, w \triangleleft, 0, \ldots, 0\right) \vdash_{M}^{*}\left(q, \triangleleft, c_{1}, \ldots, c_{k}\right)$, for some $q \in F, c_{1}, \ldots, c_{k} \in \mathbb{N}_{0}$. The language accepted by $M, L(M)$, is the set of all words accepted by $M$. Essentially, a $k$-counter machine is a $k$-pushdown machine where each pushdown has one symbol plus an end-marker. It is well known that a two counter machine is equivalent to a deterministic Turing machine [22].

In this paper, we will often restrict the counter(s) to be reversal-bounded in the sense that each counter can only reverse (i.e., change mode from non-decreasing to non-increasing and vice-versa) at most $r$ times for some given $r$. In particular, when $r=1$, the counter reverses only once, i.e., once it decrements, it can no longer increment. Note that a counter that makes $r$ reversals can be simulated by $\left\lceil\frac{r+1}{2}\right\rceil 1$-reversal-bounded counters. Closure and decidable properties of various machines augmented with reversal-bounded counters have been studied in the literature (see, e.g., 21, 23, 24, 25]). We will use the notation $\operatorname{NCM}(k, r)$ to represent $r$-reversal-bounded, $k$-counter machines, and NCM to represent all reversal-bounded multicounter machines. Machines with one unrestricted pushdown, plus reversal-bounded counters have also been studied 21]. Then, $\operatorname{NPCM}(k, r)$ are machines with one unrestricted pushdown, and $k r$-reversal-bounded counters, and NPCM are all such machines. We use ' $\mathrm{D}$ ' in place of ' $\mathrm{N}$ ' for the deterministic versions, e.g., DCM, $\operatorname{DCM}(k, r)$, DPCM, and $\operatorname{DPCM}(k, r)$. We use this notation for both the classes of machines, and the families of languages they accept.

Example 1. Consider the language $L=\left\{a^{i} b^{j} a^{i} b^{j} \mid i, j>0\right\}$. This language is neither regular nor contextfree. However, this language can be accepting by a $\operatorname{DCM}(2,1)$ machine $M$. Indeed, $M$, on input $a^{i} b^{j} a^{k} b^{l}$ reads the a's, and increases the first counter by one for each a read. Then, it reads the b's, and increases the second counter for every $b$ read. Then, it reads the second section of a's, and decreases the first counter for every a read, and verifies that it hits the final section of b's when the first counter is empty, thereby verifying that the number of a's in the first section is equal to the number in the second section. Then, it does the same with the second section of $b$ 's and the second counter. Thus, $M$ accepts if and only if $i=k$ and $j=l$.

We will also use the notation below to represent common classes of automata (and languages), where any that we have not defined are defined as in [19]: NPDA for nondeterministic pushdown automata; DPDA for deterministic pushdown automata; NCA for nondeterministic one counter machines with no reversalbound; DCA for deterministic NCAs; NFA for nondeterministic finite automata; DFA for deterministic finite automata; and DTM for deterministic Turing machines. As is well-known, NFAs, NPDAs, and DTMs, accept exactly the regular languages, context-free languages, and recursively enumerable languages, respectively.

A set $Q \subseteq \mathbb{N}_{0}^{m}$ is a linear set if there exist vectors $\overrightarrow{v_{0}}, \overrightarrow{v_{1}}, \ldots, \overrightarrow{v_{n}} \in \mathbb{N}_{0}^{m}$ such that $Q=\left\{\overrightarrow{v_{0}}+i_{1} \overrightarrow{v_{1}}+\cdots+i_{n} \overrightarrow{v_{n}} \mid\right.$ $\left.i_{1}, \ldots, i_{n} \in \mathbb{N}_{0}\right\}$. In this definition, the vector $\overrightarrow{v_{0}}$ is called the constant and $\overrightarrow{v_{1}}, \ldots, \overrightarrow{v_{n}}$ are the periods. A semilinear set is a finite union of linear sets. For semilinear sets $Q_{1}, Q_{2} \subseteq \mathbb{N}^{m}, Q_{1}+Q_{2}=\left\{v \mid v_{1}+v_{2}, v_{1} \in\right.$ $\left.Q_{1}, v_{2} \in Q_{2}\right\}$.

The Parikh map of $w \in \Sigma^{*}, \Sigma=\left\{a_{1}, \ldots, a_{m}\right\}$ is the vector $\psi(w)=\left(|w|_{a_{1}}, \ldots,|w|_{a_{m}}\right)$, and the Parikh map of $L$ is $\psi(L)=\{\psi(w) \mid w \in L\}$. For a vector $\vec{v} \in \mathbb{N}_{0}^{m}$, the inverse $\psi^{-1}(\vec{v})=\left\{w \in \Sigma^{*} \mid \psi(w)=\vec{v}\right\}$, which 
is extended to subsets of $\mathbb{N}_{0}^{m}$. A language is semilinear if its Parikh map is semilinear. The commutative closure of a language $L \subseteq \Sigma^{*}$ is $\operatorname{comm}(L)=\psi^{-1}(\psi(L))$, and a language $L$ is commutative if it is equal to its commutative closure. The family of all commutative semilinear languages is denoted by COM-SLIP, following notation developed in [26].

Let $u, v \in \Sigma^{*}$. The shuffle of $u$ and $v$, denoted $u ш v$ is the set

$$
\left\{u_{1} v_{1} u_{2} v_{2} \cdots u_{n} v_{n} \mid u_{i}, v_{i} \in \Sigma^{*}, 1 \leq i \leq n, u=u_{1} \cdots u_{n}, v=v_{1} \cdots v_{n}\right\} .
$$

This can be extended to languages $L_{1}, L_{2} \subseteq \Sigma^{*}$ by $L_{1} w L_{2}=\bigcup_{u \in L_{1}, v \in L_{2}} u ш v$. Given $u, v \in \Sigma^{*}$, there is an obvious NFA with $(|u|+1)(|v|+1)$ states accepting $u ш v$, where each state stores a position within both $u$ and $v$. This has been called the naive NFA for $u \omega v$ [17]. It was also mentioned in [17] that if $u$ and $v$ are over disjoint alphabets, then the naive NFA is a DFA.

An NFA $M=\left(Q, \Sigma, q_{0}, F, \delta\right)$ is accessible if, for each $q \in Q$, there exists $u \in \Sigma^{*}$ such that $q \in \delta\left(q_{0}, u\right)$. Also, $M$ is co-accessible if, for each $q \in Q$, there exists $u \in \Sigma^{*}$ such that $\delta(q, u) \cap F \neq \emptyset$. Lastly, $M$ is trim if it is both accessible and co-accessible, and $M$ is acyclic if $q \notin \delta(q, u)$ for every $q \in Q, u \in \Sigma^{+}$.

\section{Comparing Shuffle on Words to NFAs}

The results to follow in this section depend on the following result from [18].

Proposition 1. It is NP-complete to determine, given a DFA $M$ and words $u, v$ over an alphabet of at least two letters, if $L(M) \nsubseteq u ш v$.

First, we note that this NP-completeness can be extended to NFAs.

Corollary 2. It is NP-complete to determine, given an NFA $M$ and words $u, v$ over an alphabet of at least two letters, if $L(M) \nsubseteq u ш v$.

Proof. NP-hardness follows from Proposition 1

To show it is in NP, let $M$ be an NFA with state set $Q$. Create a nondeterministic Turing machine that guesses a word $w$ of length at most $|u v|+|Q|$, and verifies that $w \in L(M)$ and that $w \notin u ш v$ in polynomial time [18]. And indeed, $L(M) \nsubseteq \subseteq u ш v$ if and only if $L(M) \cap\left\{w|| w|\leq| u v|+| Q \mid, w \in \Sigma^{*}\right\} \nsubseteq \subseteq u ш v$, since any word longer than $|u v|+|Q|$ that is in $L(M)$ implies there is another one in $L(M)$ with length between $|u v|+1$ and $|u v|+|Q|$, which is therefore not in $u ш v$ (all words in $u ш v$ are of length $|u|+|v|$ ).

Next, the reverse inclusion of Corollary 2 will be examined. In contrast to the polynomial-time testability of $u ш v \subseteq L(M)$ when $M$ is a DFA ([16], with an alternate shorter proof appearing in Proposition 8 of this paper), testing $u ш v \nsubseteq L(M)$ is NP-complete for NFAs.

Proposition 3. It is NP-complete to determine, given an NFA $M$ and $u, v$ over an alphabet of at least two letters, whether $u ш v \nsubseteq L(M)$.

Proof. First, it is in NP, since all words in $u ш v$ are of length $|u v|$, and so a nondeterministic Turing machine can be built that nondeterministically guesses one and tests if it is not in $L(M)$ in polynomial time.

For NP-hardness, let $F$ be an instance of the 3SAT problem (a known NP-complete problem [20]) with a set of Boolean variables $X=\left\{x_{1}, \ldots, x_{p}\right\}$, and a set of clauses $\left\{c_{1}, \ldots, c_{q}\right\}$, where each clause has three literals.

If $d$ is a truth assignment, then $d$ is a function from $X$ to $\{+,-\}$ (true or false). For a variable $x$, then $x^{+}$and $x^{-}$are literals. In particular, the literal $x^{+}$is true under $d$ if and only if the variable $x$ is true under $d$. And, the literal $x^{-}$is true under $d$ if and only if the variable $x$ is false [20]. Let $y=\left\lceil\log _{2} p\right\rceil+1$, which is enough to hold the binary representation of any of $1, \ldots, p$. For an integer $i, 1 \leq i \leq p$, let $b(i)$ be the string 1 followed by the $y$-bit binary representation of $i$, followed by 1 again. 
For $1 \leq i \leq p, 1 \leq j \leq q$, let $f(i, j)$ be defined as follows, where it is a set of either one or two strings over $\{0,1\}$ :

$$
f(i, j)= \begin{cases}\{01 b(i)\} & \text { if } x_{i}^{+} \in c_{j} \\ \{10 b(i)\} & \text { if } x_{i}^{-} \in c_{j} \\ \{10 b(i), 01 b(i)\} & \text { otherwise }\end{cases}
$$

For $1 \leq j \leq q$, let $F_{j}=f(1, j) f(2, j) \cdots f(p, j)$. Therefore, $F_{j}$ is the concatenation of languages (since each $f(i, j)$ is a set of one or two strings).

We will next give the construction. Let $u=1 b(1) 1 b(2) \cdots 1 b(p)$, and let $v=0^{p}$.

Let $T=\left\{e_{1} b(1) e_{2} b(2) \cdots e_{p} b(p) \mid e_{i} \in\{10,01\}, 1 \leq i \leq p\right\}$, consisting of $2^{p}$ strings. Clearly $T \subseteq u ш v$, and also $T$ is a regular language, and a DFA $M_{T}$ can be built accepting this language in polynomial time, as with a DFA $\overline{M_{T}}$ accepting $\overline{L\left(M_{T}\right)}$.

Then, make an NFA $M^{\prime}$ accepting $\bigcup_{1 \leq j \leq q} F_{j}$. It is clear that this NFA is of polynomial size. Then, make another NFA $M^{\prime \prime}$ accepting $L\left(M^{\prime}\right) \cup L\left(\overline{M_{T}}\right)$. The following claim shows that deciding $u ш v \nsubseteq L\left(M^{\prime \prime}\right)$ is equivalent to deciding if there is a solution to the 3SAT instance.

Claim 1. The following three conditions are equivalent:

1. $u ш v \cap \overline{L\left(M^{\prime \prime}\right)} \neq \emptyset$,

2. $T \cap \overline{L\left(M^{\prime \prime}\right)} \neq \emptyset$,

3. F has a solution.

Proof. " $1 \Rightarrow 2$ ". Let $w \in u ш v \cap \overline{L\left(M^{\prime \prime}\right)}$. Then $w \notin L\left(M^{\prime \prime}\right)$, and since $L\left(\overline{M_{T}}\right) \subseteq L\left(M^{\prime \prime}\right)$, necessarily $w \in T$.

"2 $\Rightarrow 1$ ". Let $w \in T \cap \overline{L\left(M^{\prime \prime}\right)}$. But, $T \subseteq u ш v$; and so $w \in u ш v \cap \overline{L\left(M^{\prime \prime}\right)}$.

"2 $\Rightarrow 3$ ". Assume $w \in T \cap \overline{L\left(M^{\prime \prime}\right)}$. Thus, $w=e_{1} b(1) e_{2} b(2) \cdots e_{p} b(p), e_{i} \in\{10,01\}$, but $w \notin \bigcup_{1 \leq j \leq q} F_{j}$. Let $d$ be the truth assignment obtained from $w$ where

$$
d\left(x_{i}\right)= \begin{cases}+, & \text { if } e_{i}=10 \\ -, & \text { if } e_{i}=01\end{cases}
$$

for all $i, 1 \leq i \leq p$. Thus, for every $j, 1 \leq j \leq q, w \notin F_{j}$, but for all variables $x_{i}$ not in $c_{j}, e_{i} b(i)$ must be an infix of words in $F_{j}$ since $10 b(i)$ and $01 b(i)$ are both in $f(i, j)$ when $x_{i}$ is not in $c_{j}$. So one of the words encoding the (three) variables in $c_{j}$, must have $10 b(i)$ as an infix of words in $F_{j}$ where $d\left(x_{i}\right)=+$, or $01 b(i)$ as an infix of words in $F_{j}$ where $d\left(x_{i}\right)=-$, since otherwise $F_{j}$ would have as infix, for each $x_{i}$ that is a variable of $c_{j}, 01 b(i)$ if $x_{i}^{+} \in c_{j}$, and $10 b(i)$ if $x_{i}^{-} \in c_{j}$, and so $w$ would be in $F_{j}$, a contradiction. Thus, $d$ makes clause $c_{j}$ true, as is the case with every clause. Hence, $d$ is a satisfying truth assignment, and $F$ is satisfiable.

" $3 \Rightarrow 2$ ". Assume $F$ is satisfiable, hence $d$ is a satisfying truth assignment. Create

$$
w=e_{1} b(1) e_{2} b(2) \cdots e_{p} b(p),
$$

where

$$
e_{i}= \begin{cases}10, & \text { if } d\left(x_{i}\right)=+ \\ 01, & \text { if } d\left(x_{i}\right)=-\end{cases}
$$

Then $w \in T$. Also, for each $j, d$ applied to some variable, say $x_{i}$, must be in $c_{j}$, but then by the construction of $F_{j}, e_{i} b(i)$ must not be an infix of any word in $F_{j}$. Hence, $w \notin \bigcup_{1 \leq j \leq q} F_{j}, w \notin L\left(M^{\prime}\right)$, and $w \notin L\left(M^{\prime \prime}\right)$. Hence, $w \in T \cap \overline{L\left(M^{\prime \prime}\right)}$. 
Next, we examine the complexity of testing inequality between languages accepted by NFAs and words of a very simple form.

Proposition 4. It is NP-complete to test, given $a^{p}, b^{q} \in \Sigma^{*}, p, q \in \mathbb{N}_{0}$, and $M$ an NFA over $\Sigma=\{a, b\}$, whether $L(M) \neq a^{p} ш b^{q}$.

Proof. First, it is immediate that the problem is in NP, by Corollary 2 and Proposition 3 .

To show NP-hardness, the problem in Proposition 1 is used.

Given $M$, a DFA, and words $u, v$, we can construct the naive shuffle NFA $N$ for $u ш v$. The naive NFA is of polynomial size in the length of $u$ and $v$. Let $(p, q)=\left(|u v|_{a},|u v|_{b}\right)$. Then construct the naive NFA $A$ accepting $a^{p} ш b^{q}$, which is a polynomially sized DFA since $a^{p}, b^{q}$ are over disjoint alphabets. Thus, another DFA can be built accepting $\overline{L(A)}$. We can then construct an NFA $M^{\prime}$ in polynomial time which accepts $\left(a^{p} ш b^{q} \cap \overline{L(M)}\right) \cup L(N) \cup\left(L(M) \cap\left(\overline{a^{p} ш b^{q}}\right)\right)$ as $M$ is already a DFA. Also, $u ш v \subseteq a^{p} ш b^{q}$ since the latter contains all words with $p$ a's and $q$ 's's.

We will show $L(M) \subseteq u ш v$ if and only if $L\left(M^{\prime}\right)=a^{p} \omega b^{q}$.

Assume $L(M) \subseteq L(N)(=u ш v)$. Then $L(M) \cap\left(\overline{a^{p} ш b^{q}}\right)=\emptyset$ since $u ш v \subseteq a^{p} ш b^{q}$. All other words in $L\left(M^{\prime}\right)$ are in $a^{p} w b^{q}$. Thus, $L\left(M^{\prime}\right) \subseteq a^{p} w b^{q}$. Let $w \in a^{p} w b^{q}$. If $w \notin L(M)$, then $w \in L\left(M^{\prime}\right)$. If $w \in L(M)$, then $w \in L(N) \subseteq L\left(M^{\prime}\right)$, by the assumption.

Assume $L\left(M^{\prime}\right)=a^{p} w b^{q}$. Let $w \in L(M)$. Then $L(M) \cap\left(\overline{a^{p} ш b^{q}}\right)=\emptyset$ by the assumption. So, $L(M) \subseteq$ $a^{p} ш b^{q}$. Assume $w \in L(M)$ but $w \notin L(N)$. However, $w \in L\left(M^{\prime}\right)$ by the assumption, a contradiction, as $w \notin a^{p} w b^{q} \cap \overline{L(M)}$, and $w \notin L(M) \cap \overline{a^{p} w b^{q}}$, implying $w \in L(N)$.

Hence, the problem is NP-complete.

To obtain the following corollary, it only needs to be shown that the problem is in NP, which again follows from Corollary 2 and Proposition 3 .

Corollary 5. It is NP-complete to determine, given an NFA $M$ and words $u, v$ over an alphabet of size at least two, if $L(M) \neq u ш v$.

It is known that there is a polynomial-time algorithm that, given a DFA, will output two words $u$ and $v$ such that, if $L(M)$ is decomposable into the shuffle of two words, then this implies $L(M)=u ш v[16]$. Moreover, this algorithm runs in time $O(|u|+|v|)$, which is sublinear in the input $M$. This main result from [16] is as follows:

Proposition 6. Let $M$ be an acyclic, trim, non-unary DFA over $\Sigma$. Then we can determine words $u, v \in$ $\Sigma^{+}$such that, $L(M)$ has a shuffle decomposition into two words implies $L(M)=u ш v$ is the unique decomposition. This can be calculated in $O(|u|+|v|)$ time.

However, the downside to this algorithm is that it can output two strings $u$ and $v$, when $L(M)$ is not decomposable. Thus, the algorithm does not check whether $L(M)$ is decomposable, but if it is, it can find the decomposition in time usually far less than the number of states of the DFA. The decomposition also must be unique over words (this is always true when there are at least two combined letters used in words of $L(M)$ which is the purpose of the non-unary condition in the proposition statement) 14].

It is now shown that this result scales to NFAs, while remaining polynomial time complexity. The algorithm in [16] scans at most $O(|u|+|v|)$ transitions and states of the DFA from initial state towards final state. From an NFA, it becomes possible to apply the standard subset construction [19] on the NFA only by creating states and transitions for the transitions and states examined by this algorithm (thus, the NFA is never fully determinized, and only a subset of the transitions and states of the DFA are created and traversed). Because the algorithm essentially follows one "main" path from initial state to final state in the DFA, the amount of work required for NFAs is still polynomial.

Proposition 7. There is a polynomial-time complexity algorithm that, given an acyclic, non-unary NFA $M=\left(Q_{N}, \Sigma, q_{N 0}, F_{N}, \delta_{N}\right)$, can find strings $u, v \in \Sigma^{+}$, such that, $L(M)$ has a decomposition into two words implies $L(M)=u ш v$ is the unique decomposition. Moreover, this algorithm runs in time $O((|u|+$ $\left.|v|)\left|Q_{N}\right|^{2}\right)$. 
Proof sketch. Uniqueness again follows from [14].

The algorithm outputs words $u, v \in \Sigma^{+}$such that either $L(M)=u ш v$ or $M$ is not shuffle decomposable. It is based off the one described in [16], which is quite detailed, and thus not reproduced here, although we will refer to it.

In order to use the algorithm in Proposition [6 first all states that are not accessible or not co-accessible are removed. For this, a breadth-first graph search algorithm can be used to detect which states can be reached from $q_{0}$ in $O\left(\left|Q_{N}\right|^{2}\right)$ time. It also verifies that all final states reached are the same distance from the initial state, and if not, $M$ is not decomposable. Then, collapse these final states down to one state $q_{f}$ and remove all outgoing transitions, which does not change the language accepted since $M$ is acyclic. Then, check which states can be reached from $q_{f}$ following transitions in reverse using the graph search, and remove all states that cannot be reached. This results in an NFA $M_{1}=\left(Q_{1}, \Sigma, q_{1},\left\{q_{f}\right\}, \delta_{1}\right)$ that is trim and accepts $L(M)$.

Let $M_{D}=\left(Q_{D}, \Sigma, q_{D 0}, F_{D}, \delta_{D}\right)$ be the DFA obtained from $M_{1}$ via the subset construction (we do not compute this, but will refer to it). Necessarily $M_{D}$ is trim and acyclic, since $M_{1}$ is as well. Then $q_{D 0}=\left\{q_{1}\right\}, F_{D}=\left\{P \mid P \in Q_{D}, q_{f} \in P\right\}$.

We modify the algorithm of Proposition 6 as follows: In place of DFA states, we use subsets of $Q_{1}$ from $Q_{D}$ [19]. However, states and transitions are only computed as needed in the algorithm. Any time $\delta(P, a)$ is referenced in the algorithm, we first compute the deterministic transition as follows: $\delta_{D}(P, a)=$ $\bigcup_{p \in P} \delta_{1}(p, a)$, and then use this transition. Since there are at most $\left|Q_{1}\right|$ states in a subset of $Q_{1}$, any transition of $\delta_{D}$ defined on a given state and a given letter can transition to at most $\left|Q_{1}\right|$ states. Then, we can compute $\delta_{D}(P, a)$ in $O\left(\left|Q_{1}\right|^{2}\right)$ time (for each state $p \in P$, add $\delta_{1}(p, a)$ into a sorted list without duplicates). As it is making the list, it can test if this state is final by testing if $q_{f} \in \delta_{D}(P, a)$. Therefore, this algorithm inspects $O(|u|+|v|)$ states and transitions of $M_{D}$, which takes $O\left(\left|Q_{1}\right|^{2}(|u|+|v|)\right)$ time to compute using the subset construction.

\section{Testing Inclusion of the Shuffle of Languages in Another Language}

A known result involving shuffle on words is that there is a polynomial-time test to determine, given words $u, v \in \Sigma^{+}$and a DFA $M$, whether $u ш v \subseteq L(M)$ [16]. An alternate simpler proof of this result is demonstrated next, and then this proof technique will be used to extend to more general decision problems.

Proposition 8. There is a polynomial-time algorithm to determine, given $u, v \in \Sigma^{+}$, and a DFA $M$, whether or not $u ш v \subseteq L(M)$.

Proof. Clearly, $u ш v$ is a subset of $L(M)$ if and only if $L(A) \cap \overline{L(M)}=\emptyset$, where $A$ is the naive NFA accepting $u ш v$. A DFA accepting $\overline{L(M)}$ can be built in polynomial time, and the intersection is accepted by an NFA using the standard construction [19] whose emptiness can be checked in polynomial time [19].

This result will be generalized in two ways. First, instead of individual words $u$ and $v$, languages from $\operatorname{NCM}(k, r)$, for some fixed $k, r$ will be used. Moreover, instead of a DFA for the right side of the inclusion, a $\operatorname{DCM}(k, r)$ machine will be used.

Proposition 9. Let $k, r$ be any fixed integers. It is decidable, given $M_{1}, M_{2} \in \mathrm{NCM}(k, r)$ and $M_{3} \in$ $\operatorname{DCM}(k, r)$, whether $L\left(M_{1}\right) ш L\left(M_{2}\right) \subseteq L\left(M_{3}\right)$. Moreover, the decision procedure is polynomial in $n_{1}+$ $n_{2}+n_{3}$, where $n_{i}$ is the size of $M_{i}$.

Proof. First, construct from $M_{1}$ and $M_{2}$, an NCM $M_{4}$ that accepts $L\left(M_{1}\right) ш L\left(M_{2}\right)$. Clearly $M_{4}$ is an $\operatorname{NCM}(2 k, r)$, and the size of $M_{4}$ is polynomial in $n_{1}+n_{2}$.

Then, construct from $M_{3}$ a $\mathrm{DCM}(k, r)$ machine $M_{5}$ accepting the complement of $L\left(M_{3}\right)$, which can be done in polynomial time 21].

Lastly, construct from $M_{4}$ and $M_{5}$ an $\operatorname{NCM}(3 k, r)$ machine $M_{6}$ accepting $L\left(M_{4}\right) \cap L\left(M_{5}\right)$ by simulating the machines in parallel.

It is immediate that $L\left(M_{1}\right) ш L\left(M_{2}\right) \subseteq L\left(M_{3}\right)$ if and only if $L\left(M_{6}\right)=\emptyset$. Further, it has been shown that for any fixed $t, s$, it is decidable in polynomial time, given $M$ in $\operatorname{NCM}(t, s)$, whether $L(M)=\emptyset$ [27]. 
Actually, the above proposition can be made stronger. For any fixed $k, r$, the decidability of nonemptiness of $L(M)$ for an $\mathrm{NCM}(k, r)$ is in NLOG, the class of languages accepted by nondeterministic Turing machines in logarithmic space [27]. It is known that NLOG is contained in the class of languages accepted by deterministic Turing machines in polynomial time (whether or not the containment is proper is open). By careful analysis of the constructions in the proof of the above proposition, $M_{6}$, could be constructed by a logarithmic space deterministic Turing machine. Hence:

Corollary 10. Let $k, r$ be any fixed integers. The problem of deciding, given $M_{1}, M_{2} \in \mathrm{NCM}(k, r)$ and $M_{3} \in \mathrm{DCM}(k, r)$, whether $L\left(M_{1}\right) ш L\left(M_{2}\right) \subseteq L\left(M_{3}\right)$, is in NLOG.

Proposition 9 also holds if $M_{1}$ and $M_{2}$ are NFAs and $M_{3}$ is a deterministic pushdown automaton.

Proposition 11. It is decidable, given NFA $s M_{1}, M_{2}$ and $M_{3} \in$ DPDA, whether $L\left(M_{1}\right) ш L\left(M_{2}\right) \subseteq L\left(M_{3}\right)$. Moreover, the decision procedure is polynomial in $n_{1}+n_{2}+n_{3}$, where $n_{i}$ is the size of $M_{i}$.

Proof. The proof and algorithm proceeds much like the proof of Proposition 9, Given two NFAs $M_{1}, M_{2}$, another NFA $M_{4}$ that accepts $L\left(M_{1}\right) ш L\left(M_{2}\right)$ can be constructed in polynomial time. Then, for a given DPDA $M_{3}$, a DPDA $M_{5}$ can be constructed accepting its complement in polynomial time (and is of polynomial size) [28]. Also, given an NFA $M_{4}$ and a DPDA, a NPDA $M_{6}$ can be built in polynomial time accepting $L\left(M_{4}\right) \cap L\left(M_{5}\right)$. As above, $L\left(M_{1}\right) ш L\left(M_{2}\right) \subseteq L\left(M_{3}\right)$ if and only if $L(M)=\emptyset$, and emptiness is decidable in polynomial time for NPDAs [19].

In contrast to Proposition 11, the following is shown:

Proposition 12. It is undecidable, given one-state DFAs $M_{1}$ accepting $a^{*}$ and $M_{2}$ accepting $b^{*}$, and an $\operatorname{NCM}(1,1)$ machine $M_{3}$ over $\{a, b\}$, whether $L\left(M_{1}\right) \amalg L\left(M_{2}\right) \subseteq L\left(M_{3}\right)$.

Proof. Let $\Sigma=\{a, b\}$. Then $L_{1} ш L_{2}=\Sigma^{*}$. Let $M_{3} \subseteq \Sigma^{*}$ be an $\operatorname{NCM}(1,1)$ machine. Then $L_{1} ш L_{2} \subseteq L_{3}$ if and only if $L_{3}=\Sigma^{*}$. The result follows, since the universality problem for $\mathrm{NCM}(1,1)$ is undecidable [29]. The idea is the following: Given a single-tape deterministic Turing machine $Z$, we construct $M_{3}$ which, when given any input $w$, accepts if and only if $w$ does not represent a halting sequence of configurations of $Z$ on an initially blank tape (by guessing a configuration $I D_{i}$, and extracting the symbol at a nondeterministically chosen position $j$ within this configuration, storing $j$ in the counter, and then checking that the symbol in position $j$ in the next configuration $I D_{i+1}$ determined by decrementing the counter is not compatible with the next move of the DTM from $I D_{i}$; see [29]). Hence, $L\left(M_{3}\right)$ accepts the universe if and only if $Z$ does not halt. By appropriate coding, the universe can be reduced to $\{a, b\}^{*}$.

Note that the proof of Proposition[12 shows: Let $G$ be a language family such that universality is undecidable. Then it is undecidable, given one-state DFAs $M_{1}$ accepting $a^{*}$ and $M_{2}$ accepting $b^{*}$, and $L$ in $G$, whether $L\left(M_{1}\right) ш L\left(M_{2}\right) \subseteq L$.

Proposition 13. Let $L_{1}=(a+b)^{*}$ and $L_{2}=\{\lambda\}$. It is PSPACE-complete, given an NFA $M$ with input alphabet $\{a, b\}$, whether $L_{1} ш L_{2} \nsubseteq L(M)$.

Proof. Clearly, $(a+b)^{*} \Psi\{\lambda\} \nsubseteq L$ if and only if $L \neq(a+b)^{*}$. The result follows, since it is known that this question is PSPACE-complete (see, e.g., [20]).

Remark 2. In Proposition 12, if $M_{1}$ and $M_{2}$ are DFAs accepting finite languages, and $L$ is a language in any family with a decidable membership problem, then it is decidable whether $L\left(M_{1}\right) ш L\left(M_{2}\right) \subseteq L$. This is clearly true by enumerating all strings in $L\left(M_{1}\right) ш L\left(M_{2}\right)$ and testing membership in $L$.

Next, shuffle over unary alphabets is considered.

Proposition 14. It is decidable, given languages $L_{1}, L_{2}, L_{3}$ over alphabet $\{a\}$ accepted by NPCMs, whether $L_{1}$ ш $L_{2} \subseteq L_{3}$. 
PRoOF. It is known that the Parikh map of the language accepted by any NPCM is an effectively computable semilinear set (in this case over $\mathbb{N}$ ) [21] and, hence, the languages $L_{1}, L_{2}, L_{3}$ can be accepted by DFAs over a unary alphabet.

Proposition 15. It is NP-complete to decide, for an NFA $M$ over alphabet $\{a\}$, whether $a^{*} w\{\lambda\} \nsubseteq L(M)$.

Proof. Clearly, $a^{*} w\{\lambda\} \not \subset L$ if and only if $L \neq a^{*}$. The result follows, since it is known that this question is NP-complete (see, e.g., [20]).

For the case when the unary languages $L_{1}$ and $L_{2}$ are finite:

Proposition 16. It is polynomial-time decidable, given two finite unary languages $L_{1}$ and $L_{2}$ (where the lengths of the strings in $L_{1}$ and $L_{2}$ are represented in binary) and a unary language $L_{3}$ accepted by an NFA $M$, all over the same letter, whether $L_{1} ш L_{2} \subseteq L_{3}$.

Proof. Let $r$ be the sum of the cardinalities of $L_{1}$ and $L_{2}, s$ be the length of the binary representation of the longest string in $L_{1} \cup L_{2}$, and $t$ be the length of binary representation of $M$.

We represent the NFA $M$ by an $n \times n$ Boolean matrix $A_{M}$, where $n$ is the number of states of $M$, and $A_{M}(i, j)=1$ if there is a transition from state $i$ to state $j ; 0$ otherwise.

Let $x$ be the binary representation of a unary string $a^{d}$, where $d=d_{1}+d_{2}, a^{d_{1}} \in L_{1}$, and $a^{d_{2}} \in L_{2}$. To determine if $a^{d}$ is in $L_{3}$, we compute $A_{M}^{d}$ and check that for some accepting state $p$, the $(1, p)$ entry is 1. Since the computation of $A_{M}^{d}$ can be accomplished in $O(\log d)$ Boolean matrix multiplications (using the "right-to-left binary method for exponentiation" technique used to compute $x^{m}$ where $m$ is a positive integer in $O(\log m)$ multiplications, described in Section 4.6.3 of [30] $)$, and since matrix multiplication can be calculated in polynomial time, it follows that we can decide whether $L_{1} ш L_{2} \subseteq L_{3}$ in time polynomial in $r+s+t$.

However, when the alphabet of the finite languages $L_{1}, L_{2}$ is at least binary:

Proposition 17. It is NP-complete to determine, given finite languages $L_{1}$ and $L_{2}$, and an NPDA $M$ accepting $L_{3}$, whether $L_{1}$ ш $L_{2} \not L_{3}$.

Proof. NP-hardness follows from Proposition 3 . To show that it is in NP, guess a word $u \in L_{1}$, and $v \in L_{2}$, guess a word $w$ of length $|u|+|v|$, and verify that it is in $u ш v\left[18\right.$. Then, verify that $w \notin L_{3}$, which can be done in polynomial time since the membership problem for NPDAs can be solved in polynomial time.

Proposition 18. It is undecidable, given languages $L$ and $L_{1}$ accepted by 1-reversal-bounded DPDAs (resp., $\mathrm{DCA} s)$, whether $L_{1} ш\{\lambda\} \subseteq L$.

Proof. It is known that the disjointness problem for 1-reversal-bounded DPDAs (resp., DCAs) is undecidable (see, e.g., 21] ). Let $L, L_{1} \in$ DPDA. Then $L \cap L_{1}=\emptyset$ if and only if $L_{1} \subseteq \bar{L}$ if and only if $L_{1} w\{\lambda\} \subseteq \bar{L}$, and $\bar{L}$ is in DPDA as it is closed under complement.

\section{Testing Inclusion of a Language in the Shuffle of Languages}

In this section, the reverse containment is addressed. That is, given $L, L_{1}, L_{2}$, is $L \subseteq L_{1} ш L_{2}$ ? This question will depend on the language families where $L, L_{1}$, and $L_{2}$ belong.

First, for 1-reversal-bounded DPDAs, the following is immediate. The proof is identical to that of Proposition 18 .

Proposition 19. It is undecidable, given languages $L$ and $L_{1}$ accepted by 1-reversal-bounded DPDAs (resp., DCAs), whether $L \subseteq L_{1} ш\{\lambda\}$. [29].

The following proposition follows from the known undecidability of the universality problem for $\operatorname{NCM}(1,1)$ 
Proposition 20. If $L_{1} \in \operatorname{NCM}(1,1), L_{2}=\{\lambda\}$, and $L$ is the fixed regular language $\Sigma^{*}$, then it is undecidable whether $L \subseteq L_{1}$ ш $L_{2}$.

Remark 3: We are currently examining the question of whether it is undecidable, given a regular language $L$ and languages $L_{1}, L_{2}$ accepted by DPDAs (resp., DCAs, DPCMs), whether $L \subseteq L_{1} ш L_{2}$. In particular, we are interested in the simple case when $L$ and $L_{1}$ are regular and $L_{2}$ is in $\operatorname{DCM}(1,1)$.

Next, we examine some families where decidability occurs. The following is true since the shuffle of regular languages is regular, and the containment problem is decidable for regular languages.

Proposition 21. If $L_{1}, L_{2}, L$ are all regular languages, then it is decidable whether $L \subseteq L_{1} ш L_{2}$.

The following is true since the shuffle of an NPCM and an NCM is an NPCM language, and containment can be decided by using the decidable membership problem for NPCM [21].

Proposition 22. If $L_{1} \in \mathrm{NPCM}, L_{2} \in \mathrm{NCM}$, and $L$ is finite, then it is decidable whether $L \subseteq L_{1} ш L_{2}$.

Proposition 23. If $L \in \mathrm{NPCM}$, and $L_{1}, L_{2} \in \mathrm{DCM}$ over disjoint alphabets, then it is decidable whether $L \subseteq L_{1} \amalg L_{2}$.

Proof. Let $M_{1}, M_{2} \in$ DCM over disjoint alphabets, such that $L_{1}=L\left(M_{1}\right)$ and $L_{2}=L\left(M_{2}\right)$, where $M_{1}$ has $k_{1}$ counters and $M_{2}$ has $k_{2}$ counters. Then $L_{1} \amalg L_{2}$ can be accepted by a $k_{1}+k_{2}$ counter machine (by simulating $M_{1}$ on the first $k_{1}$ counters and $M_{2}$ on the last $k_{2}$ counters). Furthermore, since $L_{1}, L_{2}$ are over disjoint alphabets, $L_{1} ш L_{2}$ is in DCM as well. Then we can construct $\overline{L_{1} \amalg L_{2}}$ since DCM is closed under complement [21], and test if $L \cap \overline{L_{1} ш L_{2}}=\emptyset$, which is true if and only if $L \subseteq L_{1} ш L_{2}$.

The following can also be shown with a proof identical to Proposition 14

Proposition 24. It is decidable, given languages $L_{1}, L_{2}, L$ over alphabet $\{a\}$ accepted by NPCMs, whether: $L \subseteq L_{1} \amalg L_{2}$.

Lastly, a large family is presented for which decidability follows. These questions will be examined next for commutative languages. First, two lemmas are needed.

Lemma 25. Let $\Sigma=\left\{a_{1}, \ldots, a_{m}\right\}$. We can effectively construct, given semilinear sets $Q_{1}, Q_{2} \subseteq \mathbb{N}^{m}$, a semilinear set $Q$ such that $Q=Q_{1}+Q_{2}$. Further, we can construct a $\operatorname{DCM} M_{Q}$ to accept $\psi^{-1}(Q)$.

Proof. It is known that every COM-SLIP language is in DCM [31]. Therefore, for $i=1,2$, a DCM $M_{Q_{i}}$ can be constructed accepting $\psi^{-1}\left(Q_{i}\right)$.

Then construct an NCM $M$ to accept $\left\{a_{1}^{k_{1}} \cdots a_{m}^{k_{m}} \mid k_{1}=r_{1}+s_{1}, \ldots, k_{m}=r_{m}+s_{m}, a_{1}^{r_{1}} \cdots a_{m}^{r_{m}} \in\right.$ $\left.\psi^{-1}\left(Q_{1}\right), a_{1}^{s_{1}} \cdots a_{m}^{s_{m}} \in \psi^{-1}\left(Q_{2}\right)\right\}$ as follows: given input $w=a_{1}^{k_{1}} \cdots a_{m}^{k_{m}}, M$ reads the input and nondeterministically guesses the decompositions of the $k_{i}$ 's into $r_{i}$ 's and $s_{i}$ 's, and stores them in $2 m$ counters which we call $c_{1}, \ldots, c_{m}, d_{1}, \ldots, d_{m}$ (they store the numbers $r_{1}, \ldots, r_{m}, s_{1}, \ldots, s_{m}$ ). Then $M$ simulates the computation of $M_{Q_{1}}$ on input $a_{1}^{r_{1}} \cdots a_{m}^{r_{m}}$ (by decrementing the counters $c_{1}, \ldots, c_{m}$ which have values $r_{1}, \ldots, r_{m}$, corresponding to reading an input letter of input $M_{Q_{1}}$ ) and if $M_{Q_{1}}$ accepts, $M$ then simulates the computation of $M_{Q_{2}}$ on input $a_{1}^{s_{1}} \cdots a_{m}^{s_{m}}$ using counters $d_{1}, \ldots, d_{m}$ similarly. Then $M$ accepts if $M_{Q_{2}}$ accepts. Since NCM only accepts semilinear languages, it follows that there is a semilinear set $Q$ such that $\psi(L(M))=Q$, and hence $\psi^{-1}(Q)$ can be accepted by a DCM.

The next result follows from the definition of commutative semilinear languages and the previous lemma.

Lemma 26. Let $Q_{1}, Q_{2} \subseteq \mathbb{N}^{m}$ be semilinear sets, and $Q=Q_{1}+Q_{2}$. Then $\psi^{-1}(Q)=\psi^{-1}\left(Q_{1}\right) ш \psi^{-1}\left(Q_{2}\right)$. Moreover, $Q$ can be effectively constructed from $Q_{1}$ and $Q_{2}$.

We can now prove: 
Proposition 27. Let $\Sigma=\left\{a_{1}, \ldots, a_{m}\right\}, m \geq 1$. It is decidable, given an NPCM $M$ accepting a language $L \subseteq \Sigma^{*}$, and COM-SLIP languages $L_{1}, L_{2} \subseteq \Sigma^{*}$ (effectively semilinear), whether $L \subseteq L_{1} ш L_{2}$. Further, for $L, L_{1}, L_{2} \in \mathrm{NPCM}$, it is decidable whether $L \subseteq \operatorname{comm}\left(L_{1}\right) ш \operatorname{comm}\left(L_{2}\right)$.

Proof. The second statement follows from the first since NPCM is effectively semilinear and both $\operatorname{comm}\left(L_{1}\right)$ and $\operatorname{comm}\left(L_{2}\right)$ are in COM-SLIP.

Using the semilinear sets $Q_{1}=\psi\left(L_{1}\right), Q_{2}=\psi\left(L_{2}\right)$, then from Lemma[25] and Lemma26, we can construct a DCM $M_{1}$ accepting $L_{1} ш L_{2}$. We can then construct a DCM $M_{2}$ accepting $\overline{L\left(M_{1}\right)}$, since DCM is closed under complementation. Then we construct an NPCM $M_{3}$ (simulating $M$ and $M_{2}$ in parallel) accepting $L(M) \cap L\left(M_{2}\right)$. The result follows, since $L(M) \subseteq L_{1} ш L_{2}$ if and only if $L\left(M_{3}\right)=\emptyset$, which is decidable, since the emptiness problem for NPCMs is decidable [21].

This result generalizes from NPCM to other effectively semilinear language families, such as the nondeterministic versions of the semilinear automata models from [32].

The reverse inclusion of the above proposition is not true however, since if $L_{1}=(a+b)^{*}$ and $L_{2}=\{\lambda\}$ (trivially commutative semilinear languages), then it is undecidable, given an $\mathrm{NCM}(1,1) M$ with input alphabet $\{a, b\}$, whether $L_{1} ш L_{2} \subseteq L(M)$. This is because it is undecidable whether an $\operatorname{NCM}(1,1)$ machine is equal to $\{a, b\}^{*}$.

\section{Other Decision and Closure Properties Involving Shuffle}

The following proposition follows from the proof of Theorem 6 in [10].

Proposition 28. It is undecidable, given two languages accepted by 1-reversal-bounded DPDAs (resp., DCAs), whether their shuffle is accepted by a 1-reversal-bounded DPDA (resp., DCA).

We can show the undecidability of a related problem:

Proposition 29. It is undecidable, given two 1-reversal-bounded DPDAs $M_{1}, M_{2}$ (resp., DCAs) and a 2state DFA $M$, whether $L(M) \cap\left(L\left(M_{1}\right) \amalg L\left(M_{2}\right)\right)=\emptyset$.

Proof. Let $L_{1}$ and $L_{2}$ be accepted by 1-reversal-bounded DPDAs (resp., DCAs) over input alphabet $\Sigma$. Let $\Sigma^{\prime}=\left\{a^{\prime} \mid a \in \Sigma\right\}$. Define the homomorphism $h$ by: $h(a)=a^{\prime}$ for all $a \in \Sigma$. Let $L_{3}=\left\{w^{\prime} \mid w^{\prime}=h(w), w \in\right.$ $\left.L_{1}\right\}$. (Thus, $L_{3}$ is a primed version of $L_{1}$.) Let $L=\left\{a_{1} a_{1}^{\prime} \cdots a_{k} a_{k}^{\prime} \mid k \geq 0, a_{1}, \ldots, a_{k} \in \Sigma\right\}$. Clearly, $L$ is regular and can be accepted by a 2-state DFA. Now $L \cap\left(L_{1} ш L_{3}\right)=\emptyset$ if and only if $L_{1} \cap L_{2}=\emptyset$, which is undecidable.

Now, we consider the shuffle of languages from various families, and contrast closure results with established results on commutative languages.

Clearly, if $L_{1}, L_{2}$ are in NCM, then $L_{1} ш L_{2}$ is also in NCM. Thus, NCM is closed under shuffle. However, we have:

Proposition 30. There are languages $L_{1}, L_{2} \in \operatorname{DCM}(1,1)$ such that $L_{1} ш L_{2}$ is over a two letter alphabet, but not a context-free language (NPDA).

Proof. Let $L_{1}=\left\{a^{n} b a b^{n} a \mid n>0\right\}$ and $L_{2}=\left\{b^{m} a^{m+1} \mid m>0\right\}$. If $L=L_{1} ш L_{2}$ is a context-free language, then $L^{\prime}=L \cap\left\{a^{i} b^{j} a^{k} b^{l} a^{p} \mid i, l \geq 1, j, p>1, k=2\right\}=\left\{a^{n} b^{m+1} a^{2} b^{n} a^{m+1} \mid n, m>0\right\}$ is also a context-free language. But it is easy to show, using the Pumping Lemma, that $L^{\prime}$ is not a context-free language.

This contrasts the commutative case as $L_{1} ш L_{2}$ is over a two letter alphabet and is semilinear, but not a context-free language, but every two letter semilinear language that is commutative is a context-free language.

It is known that the commutative closure of every NPCM (or effectively semilinear language family) is a DCM language [31]. By contrast, the shuffle of two 1-reversal DPDAs can be significantly more complex, and might not even be an NPCM language, and can create non-semilinear languages. 
Proposition 31. There are languages $L_{1}, L_{2}$ accepted by 1-reversal-bounded DPDAs (resp., DCAs) such that $L_{1} ш L_{2}$ is not in NPCM.

Proof. For Part 1, let

$$
\begin{aligned}
& L_{1}=\left\{a^{i_{1}} \# a^{i_{3}} \# a^{i_{5}} \# \cdots \# a^{i_{2 n-1}} \$ a^{i_{2 n}} \# \cdots \# a^{i_{6}} \# a^{i_{4}} \# a^{i_{2}} \mid n \geq 3, i_{1}=1, i_{j+1}=\right. \\
& \left.i_{j}+1 \text { for odd } j\right\} \text {, } \\
& L_{2}=\left\{a^{i_{1}} \# a^{i_{3}} \# a^{i_{5}} \# \cdots \# a^{i_{2 n-1}} \$ a^{i_{2 n}} \# \cdots \# a^{i_{6}} \# a^{i_{4}} \# a^{i_{2}} \mid n \geq 3, i_{1}=1, i_{j+1}=\right. \\
& \left.i_{j}+1 \text { for even } j\right\} \text {. }
\end{aligned}
$$

Clearly, $L_{1}, L_{2}$ can be accepted by 1-reversal DPDAs. Let $L=L_{1} \cap L_{2}$. Then the Parikh map of $L$ is not semilinear since it has the same Parikh map as the language $\left\{a^{1} \# a^{2} \# a^{3} \# \cdots \$ a^{2 n} \mid n \geq 3\right\}$. Hence, $L$ cannot be accepted by an NPCM, since it is known that the Parikh map of any NPCM language is semilinear 21.

Now let $L_{1}, L_{2}$ be over alphabet $\Sigma=\{a, \#, \$\}$. Let $\Sigma^{\prime}=\left\{a^{\prime}, \#^{\prime}, \$^{\prime}\right\}$ be the "primed" copy of $\Sigma$. For any $x \in \Sigma^{*}$, let $x^{\prime}$ be primed version of $x$ (i.e., the symbols in $x$ are replaced by their primed copies). Let $L_{2}^{\prime}=\left\{x^{\prime} \mid x \in L_{2}\right\}$. Clearly, we can construct a 1-reversal-bounded DPDA accepting $L_{2}^{\prime}$.

Suppose $L_{3}=L_{1} ш L_{2}^{\prime}$ can be accepted by an NPCM. We can also then construct an NPCM accepting $L_{4}=L_{3} \cap\left\{s s^{\prime} \mid s \in \Sigma\right\}^{*}$. Let $h$ be a homomorphism that maps the primed symbols to $\lambda$ (i.e., they are erased) and leaves the un-primed symbols unchanged. Since NPCM languages are closed under homomorphism, $h\left(L_{4}\right)$ can also be accepted by an NPCM. This gives a contradiction, since $h\left(L_{4}\right)=L=L_{1} \cap L_{2}$ cannot be accepted by an NPCM.

The proof for Part 2 is similar, except that we modify the languages $L_{1}, L_{2}$, as follows:

$$
\begin{aligned}
& L_{1}=\left\{a^{i_{1}} \# a^{i_{2}} \# a^{i_{3}} \# a^{i_{4}} \# a^{i_{5}} \# a^{i_{6}} \# \cdots \# a^{i_{2 n-1}} \# a^{i_{2 n}} \mid \begin{array}{ll}
n \geq 3, i_{1}=1, \\
& \left.i_{j+1}=i_{j}+1 \text { for odd } j\right\}, \\
L_{2}=\left\{a^{i_{1}} \# a^{i_{2}} \# a^{i_{3}} \# a^{i_{4}} \# a^{i_{5}} \# a^{i_{6}} \# \cdots \# a^{i_{2 n-1}} \# a^{i_{2 n}} \mid \begin{array}{l}
n \geq 3, i_{1}=1, \\
\end{array} \quad i_{j+1}=i_{j}+1 \text { for even } j\right\} .
\end{array}\right.
\end{aligned}
$$

Clearly, $L_{1}, L_{2}$ can be accepted by DCAs. The rest of the proof is similar to that of Part 1 .

If in the statement of Proposition 31, one of $L_{1}, L_{2}$ is accepted by an NCM, then the proposition is no longer true, since it can be easily verified that $L_{1} ш L_{2}$ could then be accepted by an NPCM. In contrast, for NCM, there are languages accepted by deterministic counter automata $L_{1}$, such that $L_{1} ш\{\lambda\}$ is not in NCM.

We need the following lemma.

Lemma 32. $L=\left\{a^{n} b^{n} \mid n>0\right\}$ is in $\operatorname{DCM}(1,1)$, but $L^{+}$(and, hence, also $L^{*}$ ) is not in $\mathrm{NCM}$.

Proof. It is obvious that $L$ is in $\operatorname{DCM}(1,1)$. Suppose $L^{+}$can be accepted by an NCM $M$. Consider the following languages:

$$
\begin{aligned}
& L_{1}=\left\{a^{n} b^{n+1} \mid n>0\right\}^{+}\left\{a^{m} \mid m>0\right\}, \\
& L_{2}=\left\{a^{1}\right\}\left\{b^{n} a^{n+1} \mid n>0\right\}^{+} .
\end{aligned}
$$

Clearly, we can construct from $M$, NCMs $M_{1}$ and $M_{2}$ accepting $L_{1}$ and $L_{2}$, respectively. Since the family of NCM languages is closed under intersection, $L_{3}=L_{1} \cap L_{2}=\left\{a^{1} b^{2} a^{3} b^{4} \cdots a^{n} b^{n+1} a^{n+2} \mid n>0\right\}$ is also in NCM. The result follows, since the Parikh map of any NCM language is semilinear [21], but the Parikh map of $L_{3}$ is not semilinear.

Proposition 33. There is a language $L_{1}$ ( $L^{+}$in Lemma 32) accepted by a DCA such that $L_{1} ш\{\lambda\}$ is not in NCM.

Therefore, commutative closure creates much simpler languages than just the identity operation. 


\section{Conclusions and Open Problems}

We investigated the complexity and decidability of various decision problems involving the shuffle operation. In particular, we showed that the following three problems are NP-complete for a given NFA $M$, and two words $u$ and $v$ :

- Is $L(M) \nsubseteq u ш v ?$

- Is $u$ ш $v \nsubseteq \nsubseteq L(M)$ ?

- Is $L(M) \neq u ш v ?$

We showed that there is a polynomial-time algorithm to determine, for NFAs $M_{1}, M_{2}$, and a deterministic pushdown automaton $M_{3}$, whether $L\left(M_{1}\right) ш L\left(M_{2}\right) \subseteq L\left(M_{3}\right)$. The same is true when $M_{1}, M_{2}, M_{3}$ are one-way nondeterministic $l$-reversal-bounded $k$-counter machines, with $M_{3}$ being deterministic.

We also presented decidability and complexity results for testing whether given languages $L_{1}, L_{2}$, and $L$ from various languages families satisfy $L_{1}$ ш $L_{2} \subseteq L$.

We obtained some closure properties of the shuffle operation on languages. In particular, we proved:

1. There are languages $L_{1}, L_{2} \in \operatorname{DCM}(1,1)$ such that $L_{1} ш L_{2}$ is over a two letter alphabet, but is not a context-free language (NPDA).

2. There are languages $L_{1}, L_{2}$ accepted by 1-reversal-bounded DPDAs (resp., DCAs) such that $L_{1} ш L_{2}$ is not in NPCM.

3. There is a language $L_{1}$ accepted by a DCA such that $L_{1} w\{\lambda\}$ is not in NCM.

There are a number of remaining open problems including the following:

1. Is it undecidable, given a regular language $L$ and languages $L_{1}, L_{2}$ accepted by DPDAs (resp., DCAs, DPCMs), whether $L \subseteq L_{1} ш L_{2}$ ? In particular, we are interested in the simple case when $L$ and $L_{1}$ are regular and $L_{2}$ is in $\operatorname{DCM}(1,1)$.

2. Same as item (1) above except that now the question is whether $L=L_{1} ш L_{2}$.

3. What is the complexity of testing, given a DFA $M$, and words $u, v$, whether $L(M) \neq u ш v$ ?

\section{References}

[1] S. Ginsburg, E. H. Spanier, Mappings of languages by two-tape devices, J. ACM 12 (3) (1965) $423-434$.

[2] W. F. Ogden, W. E. Riddle, W. C. Round, Complexity of expressions allowing concurrency, in: Proceedings of the 5th ACM SIGACT-SIGPLAN Symposium on Principles of Programming Languages, POPL '78, ACM, New York, NY, USA, 1978, pp. 185-194.

[3] M. Domaratzki, More words on trajectories, Bulletin of the EATCS 86 (2005) 107-145.

[4] G. S. Avrunin, U. A. Buy, J. C. Corbett, Integer programming in the analysis of concurrent systems, in: Proceedings of the 3rd International Workshop on Computer Aided Verification, CAV '91, Springer-Verlag, London, UK, UK, 1992, pp. $92-102$.

[5] W. Gelade, W. Martens, F. Neven, Optimizing schema languages for XML: Numerical constraints and interleaving, SIAM Journal on Computing 38 (5) (2009) 2021-2043. doi:10.1137/070697367

[6] L. Kari, S. Konstandinidis, P. Sosík, On properties of bond-free DNA languages, Theoretical Computer Science 334 (2005) $131-159$.

[7] C. Câmpeanu, K. Salomaa, S. Vágvölgyi, Shuffle quotient and decompositions, in: W. Kuich, G. Rozenberg, A. Salomaa (Eds.), Lecture Notes in Computer Science, Vol. 2295 of 5th International Conference on Developments in Language Theory, DLT 2001, Wien, Austria, Springer, Wien, Austria, 2001, pp. 186-196.

[8] L. Kari, On language equations with invertible operations, Theoretical Computer Science 132 (1-2) (1994) 129-150.

[9] L. Kari, P. Sosík, Aspects of shuffle and deletion on trajectories, Theoretical Computer Science 332 (1-3) (2005) 47-61.

[10] H. Bordihn, M. Holzer, M. Kutrib, Some non-semi-decidability problems for linear and deterministic context-free languages, in: M. Domaratzki, A. Okhotin, K. Salomaa, S. Yu (Eds.), Proceedings of the 10th International Conference on Implementation and Application of Automata (CIAA), Vol. 3317 of Lecture Notes in Computer Science, Springer Berlin Heidelberg, 2005, pp. 68-79.

[11] J. Jȩdrzejowicz, A. Szepietowski, Shuffle languages are in P, Theoretical Computer Science 250 (2001) 31-53.

[12] S. Buss, M. Soltys, Unshuffling a square is NP-hard, Journal of Computer and System Sciences 80 (4) (2014) $766-776$. 
[13] R. Rizzi, S. Vialette, On recognizing words that are squares for the shuffle product, in: A. A. Bulatov, A. M. Shur (Eds.), Lecture Notes in Computer Science, Vol. 7913 of 8th International Computer Science Symposium in Russia, CSR 2013, Ekaterinburg, Russia, 2013, pp. 235-245.

[14] J. Berstel, L. Boasson, Shuffle factorization is unique, Theoretical Computer Science 273 (2002) $47-67$.

[15] F. Biegler, M. Daley, M. Holzer, I. McQuillan, On the uniqueness of shuffle on words and finite languages, Theoretical Computer Science 410 (2009) 3711-3724.

[16] F. Biegler, M. Daley, I. McQuillan, Algorithmic decomposition of shuffle on words, Theoretical Computer Science 454 (2012) 38-50.

[17] M. Daley, F. Biegler, I. McQuillan, On the shuffle automaton size for words, Journal of Automata, Languages and Combinatorics 15 (2010) 53-70.

[18] F. Biegler, I. McQuillan, On comparing deterministic finite automata and the shuffle of words, in: M. Holzer, M. Kutrib (Eds.), Proceedings of the 19th International Conference on Implementation and Application of Automata (CIAA), Vol. 8587 of Lecture Notes in Computer Science, Springer International Publishing, 2014, pp. 98-109.

[19] J. E. Hopcroft, J. D. Ullman, Introduction to Automata Theory, Languages, and Computation, Addison-Wesley, Reading, MA, 1979.

[20] M. R. Garey, D. S. Johnson, Computers and Intractability: A Guide to the Theory of NP-Completeness, Series of books in the mathematical sciences, W. H. Freeman and Company, New York, 1979.

[21] O. H. Ibarra, Reversal-bounded multicounter machines and their decision problems, Journal of the ACM 25 (1) (1978) $116-133$.

[22] M. L. Minsky, Recursive unsolvability of Post's problem of "tag" and other topics in theory of Turing machines, Annals of Mathematics 74 (3) (1961) 437-455.

[23] O. H. Ibarra, Automata with reversal-bounded counters: A survey, in: H. Jürgensen, J. Karhumäki, A. Okhotin (Eds.), Descriptional Complexity of Formal Systems, Vol. 8614 of Lecture Notes in Computer Science, Springer International Publishing, 2014, pp. 5-22.

[24] J. Eremondi, O. H. Ibarra, I. McQuillan, Insertion operations on deterministic reversal-bounded counter machines, in: A.-H. Dediu, E. Formenti, C. Martín-Vide, B. Truthe (Eds.), Lecture Notes in Computer Science, Vol. 8977 of 9 th International Conference on Language and Automata Theory and Applications, LATA 2015, Nice, France, 2015, pp. 200-211.

[25] J. Eremondi, O. H. Ibarra, I. McQuillan, Deletion operations on deterministic families of automata, in: R. Jain, S. Jain, F. Stephan (Eds.), Lecture Notes in Computer Science, Vol. 9076 of 12th Annual Conference on Theory and Applications of Models of Computation, TAMC 2015, Singapore, 2015, pp. 388-399.

[26] S. Crespi-Reghizzi, P. S. Pietro, Commutative languages and their composition by consensual methods, in: Proceedings 14th International Conference on Automata and Formal Languages, AFL 2014, Szeged, Hungary, 2014, pp. 216-230.

[27] E. M. Gurari, O. H. Ibarra, The complexity of decision problems for finite-turn multicounter machines, Journal of Computer and System Sciences 22 (2) (1981) 220-229.

[28] M. M. Geller, H. B. Hunt III, T. G. Szymanski, J. D. Ullman, Economy of description by parsers, dpda's, and pda's, Theoretical Computer Science 4 (1977) 143-153.

[29] B. S. Baker, R. V. Book, Reversal-bounded multipushdown machines, Journal of Computer and System Sciences 8 (3) (1974) 315-332.

[30] D. E. Knuth, Seminumerical Algorithms, 3rd Edition, Vol. 2 of The Art of Computer Programming, Addison-Wesley, Reading, Massachusetts, 1998.

[31] O. Ibarra, I. McQuillan, The effect of end-markers on counter machines and commutativity, Theoretical Computer Science TBD. doi:http://dx.doi.org/10.1016/j.tcs.2016.02.034

[32] T. Harju, O. Ibarra, J. Karhumäki, A. Salomaa, Some decision problems concerning semilinearity and commutation, Journal of Computer and System Sciences 65 (2002) 278-294. 